\title{
ON DISCRETE HESSIAN MATRIX AND CONVEX EXTENSIBILITY
}

\author{
Satoko Moriguchi \\ Advanced Institute of Industrial Technology
}

\author{
Kazuo Murota \\ University of Tokyo
}

(Received October 31, 2011)

\begin{abstract}
For functions defined on integer lattice points, discrete versions of the Hessian matrix have been considered in various contexts. In discrete convex analysis, for example, certain combinatorial properties of the discrete Hessian matrices are known to characterize $\mathrm{M}^{\mathrm{h}}$-convex and $\mathrm{L}^{\mathrm{h}}$-convex functions, which can be extended to convex functions in real variables. The relationship between convex extensibility and discrete Hessian matrices is not fully understood in general, and unfortunately, some vague or imprecise statements have been made in the literature. This note points out that the positive semidefiniteness of the discrete Hessian matrix does not imply nor is implied by convex extensibility of discrete functions.
\end{abstract}

Keywords: Discrete optimization, discrete convex function, Hessian matrix, semidefinite programming

\section{Introduction}

For functions defined on integer lattice points, discrete versions of the Hessian matrix have been considered in various contexts. In discrete convex analysis $[5,6]$, for example, certain combinatorial properties of the discrete Hessian matrices are known $[1,3]$ to characterize $\mathrm{M}^{\natural}$ convex and $\mathrm{L}^{\mathrm{h}}$-convex functions, which can be extended to convex functions in real variables. A natural definition of a discrete Hessian matrix $H_{f}(x)=\left(H_{i j}(x)\right)$ of $f: \mathbb{Z}^{n} \rightarrow \mathbb{R}$ at $x \in \mathbb{Z}^{n}$ is

$$
H_{i j}(x)=f\left(x+e_{i}+e_{j}\right)-f\left(x+e_{i}\right)-f\left(x+e_{j}\right)+f(x),
$$

where $e_{i}$ denotes the $i$ th unit vector. This is used in characterizing $\mathrm{M}^{\mathrm{b}}$-convex functions, whereas a modified definition is suitable for $\mathrm{L}^{\mathrm{h}}$-convex functions.

However, the relationship between convex extensibility and discrete Hessian matrices is not fully understood in general, and unfortunately, some vague or imprecise statements have been made in the literature. Recent papers [8-10] discuss the relationship between convex extensibility of discrete functions and the positive semidefiniteness of their Hessian matrix $H_{f}(x)$ at each point $x$. It is certainly true that a univariate discrete function $f: \mathbb{Z} \rightarrow \mathbb{R}$, with $n=1$, is convex extensible if and only if the Hessian $H_{f}(x)$, which is actually a real number, is positive semidefinite (i.e., nonnegative). But this statement is incorrect for $n \geq 2$. We point out the incorrectness of this statement by giving counterexamples in following sections.

This paper is a contribution toward clarifying the relationship between discrete Hessians and the convex extensibility of discrete functions. To be specific, this paper points out the following facts by examples:

- Even if $f: \mathbb{Z}^{2} \rightarrow \mathbb{R}$ has a convex extension to a $\mathrm{C}^{2}$ convex function, its discrete Hessian matrix is not necessarily positive semidefinite. 
- Even if $f: \mathbb{Z}^{2} \rightarrow \mathbb{R}$ has a positive semidefinite discrete Hessian matrix at every point of $\mathbb{Z}^{2}$, it is not necessarily convex extensible.

The first of the above may be rephrased as follows:

- Even if $f: \mathbb{R}^{2} \rightarrow \mathbb{R}$ is $\mathrm{C}^{2}$ convex, the discrete Hessian matrix of the function obtained as its restriction to $\mathbb{Z}^{2}$ is not positive semidefinite.

In addition, we reconsider the previous results $[1,3]$ for discrete $\mathrm{M}^{\natural}$-convex $/ \mathrm{L}^{\natural}$-convex functions to better understand the role of discrete Hessian matrices in discrete convex analysis.

\section{Convex Extensibility Does Not Imply Positive Semidefiniteness}

In this section we consider functions in discrete variables.

We first show, by giving counterexamples, that the positive semidefiniteness of the discrete Hessian matrix $H_{f}(x)$ in (1.1) is not implied by the convex extensibility of $f$.

Example 2.1. The function $f: \mathbb{Z}^{2} \rightarrow \mathbb{R}$ defined by

$$
f\left(x_{1}, x_{2}\right)=\left|x_{1}-x_{2}\right|
$$

is extensible to a convex function $\bar{f}: \mathbb{R}^{2} \rightarrow \mathbb{R}$ given by $\bar{f}\left(x_{1}, x_{2}\right)=\left|x_{1}-x_{2}\right|$. We have

$$
\begin{aligned}
& H_{11}(0,0)=f(2,0)-2 f(1,0)+f(0,0)=0, \\
& H_{12}(0,0)=H_{21}(0,0)=f(1,1)-f(1,0)-f(0,1)+f(0,0)=-2, \\
& H_{22}(0,0)=f(0,2)-2 f(0,1)+f(0,0)=0,
\end{aligned}
$$

i.e.,

$$
H_{f}(0,0)=\left[\begin{array}{rr}
0 & -2 \\
-2 & 0
\end{array}\right]
$$

This is not positive semidefinite in spite of the convex extensibility of $f$.

Whereas the convex extension of the function $f$ in Example 2.1 is piecewise linear, the function $f$ in Example 2.2 below admits a convex extension to a $\mathrm{C}^{2}$ function. Even in this smooth case, the discrete Hessian matrix is not positive semidefinite.

Example 2.2. Consider a univariate function $\varphi: \mathbb{R} \rightarrow \mathbb{R}$ defined as

$$
\varphi(t)=\left\{\begin{array}{cl}
-\frac{1}{3}|t|^{3}+\frac{4}{3}|t|^{2} & (|t| \leq 1) \\
\frac{1}{3}|t|^{2}+|t|-\frac{1}{3} & (|t| \geq 1)
\end{array}\right.
$$

which is a $\mathrm{C}^{2}$ convex function with $\varphi(0)=0, \varphi( \pm 1)=1$, and $\varphi( \pm 2)=3$. Using this $\varphi$ we define $f: \mathbb{Z}^{2} \rightarrow \mathbb{R}$ by $f\left(x_{1}, x_{2}\right)=\varphi\left(x_{1}-x_{2}\right)$, which is convex extensible to a $\mathrm{C}^{2}$ convex function $\bar{f}: \mathbb{R}^{2} \rightarrow \mathbb{R}$ given by $\bar{f}\left(x_{1}, x_{2}\right)=\varphi\left(x_{1}-x_{2}\right)$. The discrete Hessian matrix of $f$ at $(0,0)$ :

$$
H_{f}(0,0)=\left[\begin{array}{cc}
\varphi(2)-2 \varphi(1)+\varphi(0) & 2 \varphi(0)-\varphi(-1)-\varphi(1) \\
2 \varphi(0)-\varphi(-1)-\varphi(1) & \varphi(-2)-2 \varphi(-1)+\varphi(0)
\end{array}\right]=\left[\begin{array}{rr}
1 & -2 \\
-2 & 1
\end{array}\right]
$$

is not positive semidefinite in spite of the extensibility of $f$ to a $\mathrm{C}^{2}$ convex function. 


\section{Positive Semidefiniteness Does Not Imply Convex Extensibility}

Naturally, we are also concerned with the converse, that is, whether positive semidefiniteness of the discrete Hessian matrix implies convex extensibility. We construct a counterexample in two variables with the aid of semidefinite programming. In so doing we prove the following.

Theorem 3.1. There exists a function $f: \mathbb{Z}^{2} \rightarrow \mathbb{R}$ such that $f$ is not convex extensible and the discrete Hessian matrix $H_{f}(x)$ is positive semidefinite for each $x \in \mathbb{Z}^{2}$.

Our construction consists of three steps. In the first step of our construction, we construct a function on a triangular domain with the aid of semidefinite programming technique and a computer software package. In the second step, we extend the function to a function on the nonnegative orthant. Furthermore, we extend the function to a function on $\mathbb{Z}^{2}$ in the third step.

\subsection{Construction by SDP on a triangular domain}

For any finite set $A$, its cardinality is denoted by $|A|$. We use the symbols $I$ and $O$ to denote the identity matrix and the zero matrix, respectively. We denote by $\mathbb{Z}_{+}$the set of nonnegative integers.

For $c \in \mathbb{Z}_{+}$, we define

$$
\begin{aligned}
& P(c)=\left\{\left(x_{1}, x_{2}\right) \in \mathbb{Z}^{2} \mid x_{1}+x_{2} \leq c ; x_{1}, x_{2} \geq 0\right\} \\
& B(c)=\left\{\left(x_{1}, x_{2}\right) \in \mathbb{Z}^{2} \mid x_{1}+x_{2}=c ; x_{1}, x_{2} \geq 0\right\} \\
& S(c)=\left\{\left(x_{1}, x_{2}\right) \in \mathbb{Z}^{2} \mid x_{1}+x_{2} \geq c ; x_{1}, x_{2} \geq 0\right\}
\end{aligned}
$$

It is assumed that $c \geq 8$.

In this section, we construct a function $f: P(c) \rightarrow \mathbb{R}$ which satisfies the following conditions:

$$
\begin{aligned}
& H_{f}\left(x_{1}, x_{2}\right) \succeq O \quad\left(\forall\left(x_{1}, x_{2}\right) \in P(c-2)\right) \\
& f(1,1)-2 f(2,2)+f(3,3)<0 .
\end{aligned}
$$

Here the notation $X \succeq O$ for a symmetric matrix $X$ means that $X$ is positive semidefinite. The condition (3.2) implies that $f$ admits no convex extension.

\subsubsection{Construction method}

We use a SemiDefinite Programming (SDP) technique to construct a function $f$ satisfying (3.1) and (3.2) on a triangular domain. We regard (3.1) as a constraint of SDP and want to find $\left(f\left(x_{1}, x_{2}\right) \mid\left(x_{1}, x_{2}\right) \in P(c)\right)$ which minimizes $f(1,1)-2 f(2,2)+f(3,3)$ under the constraint (3.1). If the optimal objective function value is negative, its optimal solution is a desired function $f: P(c) \rightarrow \mathbb{R}$.

We first consider (3.1) as a constraint of SDP. For $f: P(c) \rightarrow \mathbb{R}$, the discrete Hessian matrix at $\left(x_{1}, x_{2}\right) \in P(c-2)$ is given by

$$
\begin{aligned}
H_{11}\left(x_{1}, x_{2}\right) & =f\left(x_{1}+2, x_{2}\right)-2 f\left(x_{1}+1, x_{2}\right)+f\left(x_{1}, x_{2}\right), \\
H_{12}\left(x_{1}, x_{2}\right) & =H_{21}\left(x_{1}, x_{2}\right) \\
& =f\left(x_{1}+1, x_{2}+1\right)-f\left(x_{1}+1, x_{2}\right)-f\left(x_{1}, x_{2}+1\right)+f\left(x_{1}, x_{2}\right), \\
H_{22}\left(x_{1}, x_{2}\right) & =f\left(x_{1}, x_{2}+2\right)-2 f\left(x_{1}, x_{2}+1\right)+f\left(x_{1}, x_{2}\right) .
\end{aligned}
$$




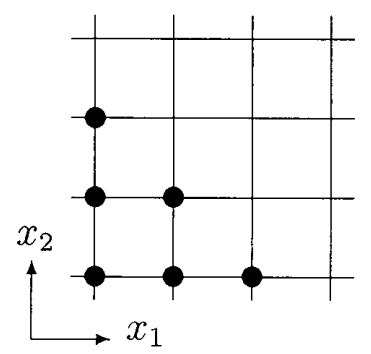

Figure 1: Six points used in the definition of $H_{f}\left(x_{1}, x_{2}\right)$

The matrix $H_{f}\left(x_{1}, x_{2}\right)$ can be written as follows:

$$
\begin{aligned}
& H_{f}\left(x_{1}, x_{2}\right)= \\
& f\left(x_{1}, x_{2}\right)\left[\begin{array}{ll}
1 & 1 \\
1 & 1
\end{array}\right]+f\left(x_{1}+1, x_{2}\right)\left[\begin{array}{rr}
-2 & -1 \\
-1 & 0
\end{array}\right]+f\left(x_{1}+2, x_{2}\right)\left[\begin{array}{ll}
1 & 0 \\
0 & 0
\end{array}\right] \\
& +f\left(x_{1}+1, x_{2}+1\right)\left[\begin{array}{ll}
0 & 1 \\
1 & 0
\end{array}\right]+f\left(x_{1}, x_{2}+1\right)\left[\begin{array}{rr}
0 & -1 \\
-1 & -2
\end{array}\right]+f\left(x_{1}, x_{2}+2\right)\left[\begin{array}{ll}
0 & 0 \\
0 & 1
\end{array}\right] .
\end{aligned}
$$

This shows that the condition $H_{f}\left(x_{1}, x_{2}\right) \succeq O$ can be formulated as an SDP constraint on six variables $f\left(x_{1}, x_{2}\right), f\left(x_{1}+1, x_{2}\right), f\left(x_{1}+2, x_{2}\right), f\left(x_{1}+1, x_{2}+1\right), f\left(x_{1}, x_{2}+1\right), f\left(x_{1}, x_{2}+2\right)$; see Figure 1. The condition $H_{f}\left(x_{1}, x_{2}\right) \succeq O$ for all $\left(x_{1}, x_{2}\right) \in P(c-2)$ can be combined as follows:

$$
\begin{gathered}
X=\sum_{\left(x_{1}, x_{2}\right) \in P(c)} F\left(x_{1}, x_{2}\right) f\left(x_{1}, x_{2}\right), \\
X \succeq O,
\end{gathered}
$$

where $F\left(x_{1}, x_{2}\right)$ is a sparse block-diagonal $(2|P(c-2)|) \times(2|P(c-2)|)$ matrix with $|P(c-2)|$ diagonal blocks corresponding to $\left(x_{1}, x_{2}\right) \in P(c-2)$.

To find $\left(f\left(x_{1}, x_{2}\right) \mid\left(x_{1}, x_{2}\right) \in P(c)\right)$ which minimizes $f(1,1)-2 f(2,2)+f(3,3)$ under (3.1), we consider the following SDP:

$$
\mathcal{P}: \begin{cases}\text { minimize } & \sum_{\left(x_{1}, x_{2}\right) \in P(c)} c\left(x_{1}, x_{2}\right) f\left(x_{1}, x_{2}\right) \\ \text { subject to } & X=\sum_{\left(x_{1}, x_{2}\right) \in P(c)} F\left(x_{1}, x_{2}\right) f\left(x_{1}, x_{2}\right), \\ & X \succeq O,\end{cases}
$$

where $\left(f\left(x_{1}, x_{2}\right) \mid\left(x_{1}, x_{2}\right) \in P(c)\right)$ is the vector of decision variables and

$$
c\left(x_{1}, x_{2}\right)=\left\{\begin{aligned}
1 & \left(\left(x_{1}, x_{2}\right)=(1,1),(3,3)\right), \\
-2 & \left(\left(x_{1}, x_{2}\right)=(2,2)\right) \\
0 & \text { (otherwise) }
\end{aligned}\right.
$$

The problem $\mathcal{P}$ is homogeneous. This means that, if there exists a feasible solution of $\mathcal{P}$ with a negative objective value, the optimal objective value is $-\infty$, that is, the problem $\mathcal{P}$ is unbounded.

To obtain a bounded SDP, we add the following constraints for normalization:

$$
\begin{gathered}
f(0,0)=1 \\
l \leq f\left(x_{1}, x_{2}\right) \leq u \quad\left(\forall\left(x_{1}, x_{2}\right) \in P(c)\right)
\end{gathered}
$$


with $l<1<u$. It is noted that the resulting SDP has a feasible solution, e.g., a constant function $f\left(x_{1}, x_{2}\right)=1$ for all $\left(x_{1}, x_{2}\right) \in P(c)$.

Remark 3.1. When we solve SDP by a computer software, we cannot avoid round-off errors. In practice, it is useful to replace the constraint $X \succeq O$ by $X-\sigma I \succeq O$ with some $\sigma>0$, and to round the approximate optimal variable vector computed by the software to an integer vector, which is to be checked for feasibility.

Remark 3.2. Here is a remark related to our choice $f(1,1)-2 f(2,2)+f(3,3)$ in $(3.2)$. The positive semidefiniteness of the discrete Hessian matrix $H_{f}\left(x_{1}, x_{2}\right)$ implies (discrete) convexity along a line with $x_{1}+x_{2}$ constant:

$$
f\left(x_{1}+2, x_{2}\right)-2 f\left(x_{1}+1, x_{2}+1\right)+f\left(x_{1}, x_{2}+2\right) \geq 0
$$

which can be shown as follows. By $H_{f}\left(x_{1}, x_{2}\right) \succeq O$ we have $H_{11} \geq 0$ and $H_{22} \geq 0$. We also have

$$
\operatorname{det} H_{f}\left(x_{1}, x_{2}\right)=H_{11} H_{22}-H_{12}^{2} \geq 0
$$

from which follows that

$$
H_{12} \leq\left|H_{12}\right| \leq \sqrt{H_{11} H_{22}} \leq \frac{1}{2}\left(H_{11}+H_{22}\right)
$$

By substituting the expressions of $H_{12}, H_{11}$ and $H_{22}$, we obtain (3.5). In view of (3.5) we search for the failure of convexity along a line with $x_{1}-x_{2}$ constant.

Remark 3.3. Our construction method by SDP is valid not only for the case $n=2$, but also for an arbitrary dimension $n$.

\subsubsection{Numerical result}

We here show a concrete numerical result of $f: P(c) \rightarrow \mathbb{R}$ satisfying (3.1) and (3.2) for $c=10$. We solve SDP $\mathcal{P}$ with (3.3) and (3.4) with $l=-100$ and $u=100$ by SDPA [11], which is a computer software package for solving SDP while extensively utilizing sparseness of the matrices; see [4] for details. We also put $\sigma=3$ in $X-\sigma I \succeq O$ to construct an integer-valued $f$ from the output of SDPA; see Remark 3.1.

By rounding an approximate optimal variable vector in the output of SDPA to an integer vector, we get $f\left(x_{1}, x_{2}\right)\left(\left(x_{1}, x_{2}\right) \in P(10)\right)$ as follows:

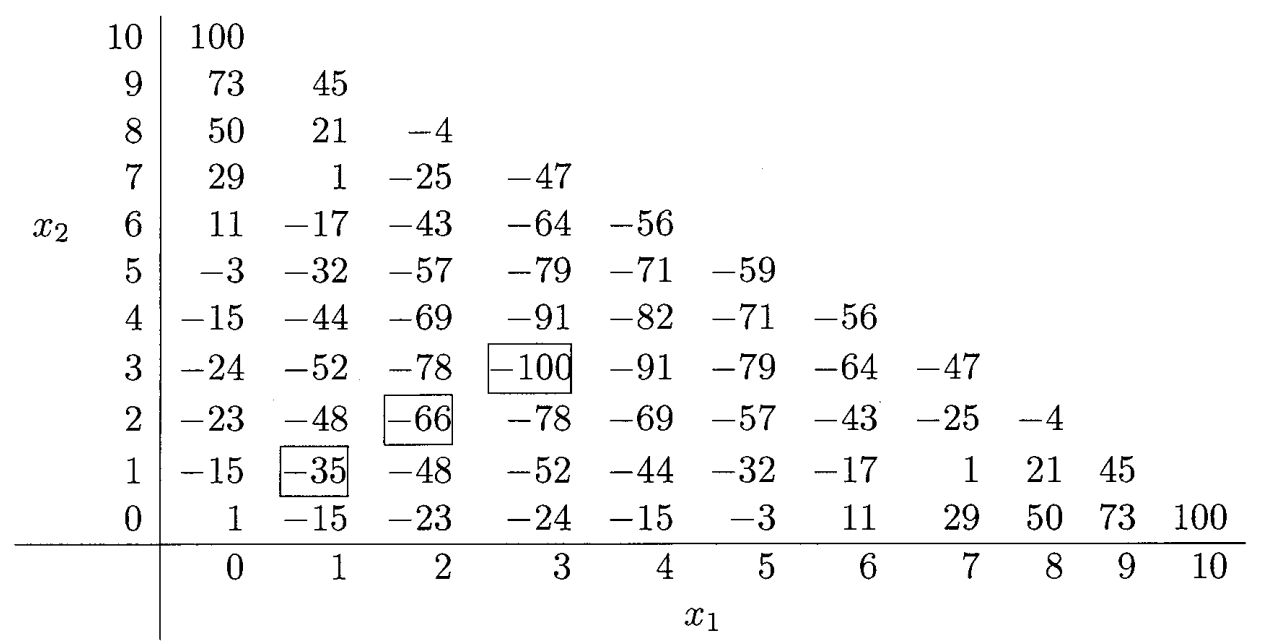

This function $f: P(c) \rightarrow \mathbb{R}$ serves as an example that the positive semidefiniteness of the discrete Hessian matrix does not imply convex extensibility. We can see a failure of midpoint convexity in

$$
f(1,1)-2 f(2,2)+f(3,3)=-35+2 \times 66-100=-3
$$


and the positive semidefiniteness of the discrete Hessian matrix $H_{f}\left(x_{1}, x_{2}\right)$ for all $\left(x_{1}, x_{2}\right) \in$ $P(8)$ can be verified.

\subsection{Extension to a function on the nonnegative orthant $\mathbb{Z}_{+}^{2}$}

In this section, we construct a function $\tilde{f}: \mathbb{Z}_{+}^{2} \rightarrow \mathbb{R}$ which satisfies the following conditions:

$$
\begin{aligned}
& H_{\tilde{f}}\left(x_{1}, x_{2}\right) \succeq O \quad\left(\forall\left(x_{1}, x_{2}\right) \in \mathbb{Z}_{+}^{2}\right), \\
& \tilde{f}(1,1)-2 \tilde{f}(2,2)+\tilde{f}(3,3)<0 .
\end{aligned}
$$

Let $f$ be the function on $P(c)$ constructed in Section 3.1.2. To extend it to $f: \mathbb{Z}_{+}^{2} \rightarrow \mathbb{R}$, we define the function value for $\left(x_{1}, x_{2}\right) \in S(c+1)$ by

$$
f\left(x_{1}, x_{2}\right)=\alpha\left(x_{1}+x_{2}\right)+\beta
$$

with arbitrary $\alpha$ and $\beta$. Then we have

$$
H_{f}\left(x_{1}, x_{2}\right)=O \quad\left(\forall\left(x_{1}, x_{2}\right) \in S(c+1)\right)
$$

and $H_{f}\left(x_{1}, x_{2}\right)$ is known to be positive semidefinite unless $\left(x_{1}, x_{2}\right) \in B(c-1) \cup B(c)$.

To take care of the case $\left(x_{1}, x_{2}\right) \in B(c-1) \cup B(c)$, we now define the following functions $g$ and $h$ with $a>0$ :

$$
\begin{gathered}
g\left(x_{1}, x_{2}\right)=a\left(x_{1}-x_{2}\right)^{2} \\
h\left(x_{1}, x_{2}\right)= \begin{cases}0 & \left(\left(x_{1}, x_{2}\right) \in \mathbb{Z}_{+}^{2}\right), \\
\left.\left.a\left(x_{1}+x_{2}-c\right)_{2}\right) \in P(c)\right), & \left(\left(x_{1}, x_{2}\right) \in S(c+1)\right) .\end{cases}
\end{gathered}
$$

It is noted that

$$
\begin{aligned}
& g(1,1)=g(2,2)=g(3,3)=0 \\
& h(1,1)=h(2,2)=h(3,3)=0
\end{aligned}
$$

and the discrete Hessian matrices of $g$ and $h$ are given as follows:

$$
\begin{gathered}
H_{g}\left(x_{1}, x_{2}\right)=a\left[\begin{array}{rr}
2 & -2 \\
-2 & 2
\end{array}\right] \quad\left(\left(x_{1}, x_{2}\right) \in \mathbb{Z}_{+}^{2}\right), \\
H_{h}\left(x_{1}, x_{2}\right)=\left\{\begin{aligned}
& {\left[\begin{array}{ll}
0 & 0 \\
0 & 0
\end{array}\right] }\left(\left(x_{1}, x_{2}\right) \in P(c-2)\right), \\
& a\left[\begin{array}{ll}
1 & 1 \\
1 & 1 \\
2 & 2 \\
2 & 2
\end{array}\right]\left(\left(x_{1}, x_{2}\right) \in B(c-1)\right), \\
&\left(\left(x_{1}, x_{2}\right) \in S(c)\right) .
\end{aligned}\right.
\end{gathered}
$$

Lemma 3.1. The conditions (3.7) and (3.8) hold for $\tilde{f}=f+g+h$ with a sufficiently large $a$.

Proof. We can see the failure of midpoint convexity (3.8) easily from (3.6), (3.10) and (3.11). We here prove the positive semidefiniteness of the discrete Hessian matrix (3.7). Note first that

$$
H_{\tilde{f}}=H_{f}+H_{g}+H_{h}
$$

We consider the following four cases: 
(i) For $\left(x_{1}, x_{2}\right) \in P(c-2)$ : From $H_{f} \succeq O$ and $H_{h}=O$, we have

$$
H_{\tilde{f}}=H_{f}+a\left[\begin{array}{rr}
2 & -2 \\
-2 & 2
\end{array}\right] \succeq O
$$

(ii) For $\left(x_{1}, x_{2}\right) \in B(c-1)$ : Although $H_{f}$ is not necessarily positive semidefinite, using a sufficiently large $a$, the positive semidefiniteness

$$
H_{\tilde{f}}=H_{f}+a\left[\begin{array}{rr}
3 & -1 \\
-1 & 3
\end{array}\right] \succeq O
$$

holds for all $\left(x_{1}, x_{2}\right) \in B(c-1)$. It is noted that such $a$ exists since $B(c-1)$ is a finite set.

(iii) For $\left(x_{1}, x_{2}\right) \in B(c)$ : Although $H_{f}$ is not necessarily positive semidefinite, using a sufficiently large $a$, the positive semidefiniteness

$$
H_{\tilde{f}}=H_{f}+a\left[\begin{array}{ll}
4 & 0 \\
0 & 4
\end{array}\right] \succeq O
$$

holds for any $\left(x_{1}, x_{2}\right) \in B(c)$. It is noted that such $a$ exists since $B(c)$ is a finite set.

(iv) For $\left(x_{1}, x_{2}\right) \in S(c+1)$ : From $H_{f}=O$, we have

$$
H_{\tilde{f}}=H_{g}+H_{h}=a\left[\begin{array}{ll}
4 & 0 \\
0 & 4
\end{array}\right] \succeq O .
$$

This completes the proof of Lemma 3.1.

\subsection{Extension to a function on $\mathbb{Z}^{2}$}

In this section, we construct a function $\hat{f}: \mathbb{Z}^{2} \rightarrow \mathbb{R}$ which satisfies the following conditions:

$$
\begin{aligned}
& H_{\hat{f}}\left(x_{1}, x_{2}\right) \succeq O \quad\left(\forall\left(x_{1}, x_{2}\right) \in \mathbb{Z}^{2}\right), \\
& \hat{f}(1,1)-2 \hat{f}(2,2)+\hat{f}(3,3)<0 .
\end{aligned}
$$

Lemma 3.2. Suppose that a function $f: \mathbb{Z}_{+}^{2} \rightarrow \mathbb{R}$ satisfies the following conditions:

$$
\begin{aligned}
& H_{f}\left(x_{1}, x_{2}\right) \succeq O \quad\left(\forall\left(x_{1}, x_{2}\right) \in \mathbb{Z}_{+}^{2}\right), \\
& \sup _{x_{1} \in \mathbb{Z}_{+}}\left(f\left(x_{1}, 0\right)-f\left(x_{1}, 1\right)\right)<+\infty, \quad \sup _{x_{2} \in \mathbb{Z}_{+}}\left(f\left(0, x_{2}\right)-f\left(1, x_{2}\right)\right)<+\infty .
\end{aligned}
$$

Choose a constant $b$ as

$$
b \geq \max \left\{\sup _{x_{1} \in \mathbb{Z}_{+}}\left(f\left(x_{1}, 0\right)-f\left(x_{1}, 1\right)\right), \sup _{x_{2} \in \mathbb{Z}_{+}}\left(f\left(0, x_{2}\right)-f\left(1, x_{2}\right)\right)\right\}
$$

and define a function $\hat{f}: \mathbb{Z}^{2} \rightarrow \mathbb{R}$ as

$$
\hat{f}\left(x_{1}, x_{2}\right)= \begin{cases}f\left(x_{1}, x_{2}\right) & \left(x_{1} \geq 0, x_{2} \geq 0\right) \\ f\left(x_{1}, 0\right)-b x_{2} & \left(x_{1} \geq 0, x_{2} \leq 0\right) \\ f\left(0, x_{2}\right)-b x_{1} & \left(x_{1} \leq 0, x_{2} \geq 0\right) \\ f(0,0)-b\left(x_{1}+x_{2}\right) & \left(x_{1} \leq 0, x_{2} \leq 0\right)\end{cases}
$$

Then the condition (3.12) is satisfied. 
Proof. From condition (3.14), we have

$$
\begin{array}{ll}
f\left(x_{1}+2,0\right)-2 f\left(x_{1}+1,0\right)+f\left(x_{1}, 0\right) \geq 0 & \left(x_{1} \geq 0\right) \\
f\left(0, x_{2}+2\right)-2 f\left(0, x_{2}+1\right)+f\left(0, x_{2}\right) \geq 0 & \left(x_{2} \geq 0\right) .
\end{array}
$$

We check the positive semidefiniteness (3.12) in the following cases:

(i) For $x_{1} \geq 0, x_{2} \geq 0$ : We have $H_{\hat{f}}\left(x_{1}, x_{2}\right)=H_{f}\left(x_{1}, x_{2}\right) \succeq O$.

(ii) For $x_{1} \geq 0, x_{2}=-1$ : We have

$$
\begin{aligned}
& H_{\hat{f}}\left(x_{1},-1\right) \\
& =\left[\begin{array}{cc}
f\left(x_{1}+2,0\right)-2 f\left(x_{1}+1,0\right)+f\left(x_{1}, 0\right) & 0 \\
0 & f\left(x_{1}, 1\right)-f\left(x_{1}, 0\right)+b
\end{array}\right] \succeq O .
\end{aligned}
$$

(iii) For $x_{1} \geq 0, x_{2} \leq-2$ : We have

$$
H_{\hat{f}}\left(x_{1}, x_{2}\right)=\left[\begin{array}{cc}
f\left(x_{1}+2,0\right)-2 f\left(x_{1}+1,0\right)+f\left(x_{1}, 0\right) & 0 \\
0 & 0
\end{array}\right] \succeq O .
$$

(iv) For $x_{1}=-1, x_{2}=-1$ : We have

$$
H_{\hat{f}}(-1,-1)=\left[\begin{array}{cc}
f(1,0)-f(0,0)+b & 0 \\
0 & f(0,1)-f(0,0)+b
\end{array}\right] \succeq O .
$$

(v) For $x_{1}=-1, x_{2} \leq-2$ : We have

$$
H_{\hat{f}}\left(-1, x_{2}\right)=\left[\begin{array}{cc}
f(1,0)-f(0,0)+b & 0 \\
0 & 0
\end{array}\right] \succeq O .
$$

(vi) For $x_{1} \leq-2, x_{2} \leq-2$ : We have $H_{\hat{f}}\left(x_{1}, x_{2}\right)=O$.

(vii) In the above we have dealt with the case where $x_{1} \geq x_{2}$. The remaining case with $x_{1} \leq x_{2}$ follows from symmetry.

Lemma 3.3. For the function $\tilde{f}: \mathbb{Z}_{+}^{2} \rightarrow \mathbb{R}$ constructed in Lemma 3.1, we have

$$
\begin{array}{ll}
\tilde{f}\left(x_{1}, 0\right)-\tilde{f}\left(x_{1}, 1\right)=2 a(c-1)-\alpha & \left(x_{1} \geq c+1\right), \\
\tilde{f}\left(0, x_{2}\right)-\tilde{f}\left(1, x_{2}\right)=2 a(c-1)-\alpha & \left(x_{2} \geq c+1\right) .
\end{array}
$$

Therefore, the function $\tilde{f}$ satisfies the condition (3.15) and, the condition (3.16) holds for $b$ with

$$
b \geq \max \left\{\max _{0 \leq x_{1} \leq c}\left(\tilde{f}\left(x_{1}, 0\right)-\tilde{f}\left(x_{1}, 1\right)\right), \max _{0 \leq x_{2} \leq c}\left(\tilde{f}\left(0, x_{2}\right)-\tilde{f}\left(1, x_{2}\right)\right), 2 a(c-1)-\alpha\right\} .
$$

Proof. For $x_{1} \geq c+1$, we have

$$
\begin{aligned}
& \tilde{f}\left(x_{1}, 0\right)-\tilde{f}\left(x_{1}, 1\right) \\
& =\left[\left(\alpha x_{1}+\beta\right)-\left(\alpha\left(x_{1}+1\right)+\beta\right)\right]+\left[g\left(x_{1}, 0\right)-g\left(x_{1}, 1\right)\right]+\left[h\left(x_{1}, 0\right)-h\left(x_{1}, 1\right)\right] \\
& =-\alpha+a\left[x_{1}^{2}-\left(x_{1}-1\right)^{2}\right]+a\left[\left(x_{1}-c\right)^{2}-\left(x_{1}+1-c\right)^{2}\right] \\
& =2 a(c-1)-\alpha .
\end{aligned}
$$

We can calculate $\tilde{f}\left(0, x_{2}\right)-\tilde{f}\left(1, x_{2}\right)$ similarly. 
The function $\hat{f}: \mathbb{Z}^{2} \rightarrow \mathbb{R}$ constructed in Lemma 3.2 with $f=\tilde{f}$ in Lemma 3.1 satisfies (3.12) and (3.13). The positive semidefiniteness (3.12) follows from Lemmas 3.1, 3.2 and 3.3 , whereas the failure of midpoint convexity (3.13) follows easily from the definition of $\hat{f}$ and Lemma 3.1. Hence this function $\hat{f}$ serves as the function $f$ in Theorem 3.1.

The construction method presented in the above is summarized as follows:

1. We construct a function $f: P(c) \rightarrow \mathbb{R}$ on the triangular domain $P(c)$ with the aid of SDP. The positive semidefiniteness of the discrete Hessian matrix holds for $\left(x_{1}, x_{2}\right) \in$ $P(c-2)$. The midpoint convexity fails at $\left(x_{1}, x_{2}\right)=(1,1),(2,2),(3,3)$.

2. We extend $f$ to a function $f: \mathbb{Z}_{+}^{2} \rightarrow \mathbb{R}$ on the nonnegative orthant by (3.9). The positive semidefiniteness of the discrete Hessian matrix holds except at $\left(x_{1}, x_{2}\right) \in B(c-1) \cup B(c)$.

3. We define $\tilde{f}: \mathbb{Z}_{+}^{2} \rightarrow \mathbb{R}$ by $\tilde{f}=f+g+h$. The positive semidefiniteness of the discrete Hessian matrix holds for $\left(x_{1}, x_{2}\right) \in \mathbb{Z}_{+}^{2}$. The midpoint convexity fails at $\left(x_{1}, x_{2}\right)=$ $(1,1),(2,2),(3,3)$.

4. We extend $\tilde{f}$ to a function $\hat{f}: \mathbb{Z}^{2} \rightarrow \mathbb{R}$ by (3.17). The positive semidefiniteness of the discrete Hessian matrix holds for $\left(x_{1}, x_{2}\right) \in \mathbb{Z}^{2}$. The midpoint convexity fails at $\left(x_{1}, x_{2}\right)=(1,1),(2,2),(3,3)$.

Remark 3.4. In Theorem 3.1 the function $f$ can be chosen to be integer-valued. To see this note that the function $f$ on $P(c)$ satisfying (3.1) and (3.2) computed by SDP is integervalued and the parameters $\alpha, \beta, a$, and $b$ can be chosen to be integers. Then the resulting function $\hat{f}$ is integer-valued.

\section{4. $\mathrm{M}^{\mathrm{h}}$-Convex/ $\mathrm{L}^{\mathrm{h}}-$ Convex Functions}

In this section, we deal with $\mathrm{M}^{\natural}$-convex and $\mathrm{L}^{\mathrm{h}}$-convex functions, which play central roles in discrete convex analysis $[5,6]$. Our objective here is to discuss the significance of the previous results on discrete Hessian matrices of $\mathrm{M}^{\mathrm{h}}$-convex and $\mathrm{L}^{\mathrm{b}}$-convex functions in the light of the examples we have seen in Sections 2 and 3. Both $M^{\natural}$ - and $L^{\natural}$-convex functions are convex extensible, and their characterizations in terms of discrete Hessians (Theorems 4.1 and 4.2 below) are combinatorial conditions that are stronger than positive semidefiniteness.

For a vector $x \in \mathbb{Z}^{n}$ and an element $i \in\{1,2, \ldots, n\}, x_{i}$ means the component of $x$ with index $i$. We write the positive and negative supports of a vector $x$ by

$$
\begin{aligned}
& \operatorname{supp}^{+}(x)=\left\{i \in\{1,2, \ldots, n\} \mid x_{i}>0\right\}, \\
& \operatorname{supp}^{-}(x)=\left\{i \in\{1,2, \ldots, n\} \mid x_{i}<0\right\} .
\end{aligned}
$$

We write $\mathbf{1}=(1,1, \ldots, 1) \in \mathbb{Z}^{n}$ and $\mathbf{0}=(0,0, \ldots, 0) \in \mathbb{Z}^{n}$. For a function $f: \mathbb{Z}^{n} \rightarrow$ $\mathbb{R} \cup\{+\infty\}$, the effective domain, denoted as $\operatorname{dom} f$, is defined to be the set of $x \in \mathbb{Z}^{n}$ for which $f(x)$ is finite.

\section{1. $\mathrm{M}^{\mathrm{b}}$-convex functions}

A function $f: \mathbb{Z}^{n} \rightarrow \mathbb{R} \cup\{+\infty\}$ is said to be $\mathrm{M}^{\natural}$-convex if it satisfies

$\left(\mathbf{M}^{h}-\mathbf{E X C}\right) \forall x, y \in \operatorname{dom} f, \forall i \in \operatorname{supp}^{+}(x-y), \exists j \in \operatorname{supp}^{-}(x-y) \cup\{0\}$ such that

$$
f(x)+f(y) \geq f\left(x-e_{i}+e_{j}\right)+f\left(y+e_{i}-e_{j}\right),
$$

where $e_{0}=\mathbf{0}$.

An $\mathrm{M}^{\mathrm{k}}$-convex function $f: \mathbb{Z}^{n} \rightarrow \mathbb{R} \cup\{+\infty\}$ can be extended to a convex function $\bar{f}: \mathbb{R}^{n} \rightarrow$ $\mathbb{R} \cup\{+\infty\}$.

We now consider the Hessian matrix. $\mathrm{M}^{\natural}$-convex functions can be characterized by a certain combinatorial property of the discrete Hessian matrix, as follows. 
Theorem $4.1([1,6])$. A function $f: \mathbb{Z}^{n} \rightarrow \mathbb{R}$ is $\mathrm{M}^{\natural}$-convex if and only if the discrete Hessian matrix $H_{f}(x)=\left(H_{i j}(x)\right)$ in (1.1) satisfies the following conditions for each $x \in \mathbb{Z}^{n}$ :

$$
\begin{array}{ll}
H_{i j}(x) \geq \min \left\{H_{i k}(x), H_{j k}(x)\right\} & \text { if }\{i, j\} \cap\{k\}=\emptyset, \\
H_{i j}(x) \geq 0 & \text { for any }(i, j) .
\end{array}
$$

It is known that a symmetric matrix satisfying the conditions (4.1) and (4.2) above is necessarily positive semidefinite.

Example 4.1. The function $f: \mathbb{Z}^{2} \rightarrow \mathbb{R}$ defined as $f\left(x_{1}, x_{2}\right)=\varphi\left(x_{1}+x_{2}\right)$ with a univariate convex function $\varphi: \mathbb{R} \rightarrow \mathbb{R}$ is $\mathrm{M}^{\mathrm{b}}$-convex. The discrete Hessian matrix of $f$ at $\left(x_{1}, x_{2}\right)$ is given by

$$
H_{f}\left(x_{1}, x_{2}\right)=\left(\varphi\left(x_{1}+x_{2}+2\right)-2 \varphi\left(x_{1}+x_{2}+1\right)+\varphi\left(x_{1}+x_{2}\right)\right)\left[\begin{array}{ll}
1 & 1 \\
1 & 1
\end{array}\right],
$$

which is positive semidefinite, since $\varphi\left(x_{1}+x_{2}+2\right)-2 \varphi\left(x_{1}+x_{2}+1\right)+\varphi\left(x_{1}+x_{2}\right) \geq 0$ by the assumed convexity of $\varphi$. For the function $\varphi(t)$ in (2.1), in particular, we have

$$
H_{f}(0,0)=\left[\begin{array}{ll}
1 & 1 \\
1 & 1
\end{array}\right]
$$

Compare this with Example 2.2, which shows that the discrete Hessian matrix of $f\left(x_{1}, x_{2}\right)=$ $\varphi\left(x_{1}-x_{2}\right)$ with $\varphi(t)$ in $(2.1)$ is not positive semidefinite; note that (4.2) is not satisfied.

As we have repeatedly seen, convex extensibility alone does not imply positive semidefiniteness of the discrete Hessian (1.1). On the other hand, $\mathrm{M}^{\natural}$-convexity, which is a combinatorial convexity concept, does imply both convex extensibility and positive semidefiniteness of the discrete Hessian via (4.1) and (4.2).

\section{2. $\mathrm{L}^{\mathrm{h}}$-convex functions}

A function $f: \mathbb{Z}^{n} \rightarrow \mathbb{R} \cup\{+\infty\}$ is called $\mathrm{L}^{\mathrm{h}}$-convex if it satisfies the following discrete midpoint convexity:

$$
f(x)+f(y) \geq f\left(\left\lceil\frac{x+y}{2}\right\rceil\right)+f\left(\left\lfloor\frac{x+y}{2}\right\rfloor\right) \quad\left(x, y \in \mathbb{Z}^{n}\right),
$$

where $\left\lceil\frac{x+y}{2}\right\rceil$ and $\left\lfloor\frac{x+y}{2}\right\rfloor$ denote, respectively, the integer vectors obtained from $\frac{x+y}{2}$ by componentwise round-up and round-down to the nearest integers. An $\mathrm{L}^{\mathrm{b}}$-convex function $f: \mathbb{Z}^{n} \rightarrow \mathbb{R} \cup\{+\infty\}$ can be extended to a convex function $\bar{f}: \mathbb{R}^{n} \rightarrow \mathbb{R} \cup\{+\infty\}$.

We now consider the Hessian matrix.

Example 4.2. The function $f: \mathbb{Z}^{2} \rightarrow \mathbb{R}$ defined as $f\left(x_{1}, x_{2}\right)=\varphi\left(x_{1}-x_{2}\right)$ with a univariate convex function $\varphi: \mathbb{R} \rightarrow \mathbb{R}$ is $L^{\mathrm{b}}$-convex. Recall Example 2.2, which shows that the discrete Hessian matrix (1.1) of $f\left(x_{1}, x_{2}\right)=\varphi\left(x_{1}-x_{2}\right)$ with $\varphi(t)$ in $(2.1)$ is not positive semidefinite at $\left(x_{1} ; x_{2}\right)=(0,0)$.

Example 4.3. The function $f: \mathbb{Z}^{4} \rightarrow \mathbb{R}$ defined by

$$
f(x)=\max \left\{x_{1}+x_{2}, x_{3}+x_{4}, x_{1}+x_{3}+1, x_{1}+x_{4}+1, x_{2}+x_{3}+1, x_{2}+x_{4}+1\right\}
$$

is $\mathrm{L}^{\mathrm{h}}$-convex [5]. The discrete Hessian matrix (1.1) at $\left(x_{1}, x_{2}, x_{3}, x_{4}\right)=(0,0,0,0)$ is

$$
H_{f}(0,0,0,0)=\left[\begin{array}{rrrr}
0 & -1 & 0 & 0 \\
-1 & 0 & 0 & 0 \\
0 & 0 & 0 & -1 \\
0 & 0 & -1 & 0
\end{array}\right]
$$

which is not positive semidefinite. 
The above examples demonstrate that the natural definition (1.1) of the discrete Hessian matrix is not amenable to $\mathrm{L}^{\mathrm{h}}$-convexity. An alternative possibility is pursued in [3], which turned out to be suitable for $\mathrm{L}^{\mathrm{b}}$-convexity.

For $f: \mathbb{Z}^{n} \rightarrow \mathbb{R}, x \in \mathbb{Z}^{n}$, and $i, j \in\{1, \ldots, n\}$ with $i \neq j$, we first define

$$
\begin{aligned}
\eta_{i j}(x) & =-f\left(x+e_{i}+e_{j}\right)+f\left(x+e_{i}\right)+f\left(x+e_{j}\right)-f(x), \\
\eta_{i}(x) & =f(x)+f\left(x+1+e_{i}\right)-f(x+1)-f\left(x+e_{i}\right) .
\end{aligned}
$$

Then we define a symmetric matrix $\tilde{H}_{f}(x)=\left(\tilde{H}_{i j}(x) \mid i, j=1, \ldots, n\right)$ by

$$
\tilde{H}_{i j}(x)=-\eta_{i j}(x) \quad(i \neq j), \quad \tilde{H}_{i i}(x)=\eta_{i}(x)+\sum_{j \neq i} \eta_{i j}(x)
$$

as a variant of the discrete Hessian matrix. The modified discrete Hessian matrix gives a characterization of $\mathrm{L}^{\mathrm{q}}$-convex functions, as follows.

Theorem $4.2([3])$. A function $f: \mathbb{Z}^{n} \rightarrow \mathbb{R}$ is $L^{\mathrm{h}}$-convex if and only if the modified discrete Hessian matrix $\tilde{H}_{f}(x)=\left(\tilde{H}_{i j}(x)\right)$ in (4.4) satisfies the following conditions for each $x \in \mathbb{Z}^{n}$ :

$$
\begin{aligned}
& \tilde{H}_{i j}(x) \leq 0 \quad(i, j \in\{1, \ldots, n\}, i \neq j), \\
& \sum_{j=1}^{n} \tilde{H}_{i j}(x) \geq 0 \quad(i \in\{1, \ldots, n\}) .
\end{aligned}
$$

It is known that a symmetric matrix satisfying the conditions (4.5) and (4.6) above is necessarily positive semidefinite.

Example 4.4. For the $\mathrm{L}^{\mathrm{h}}$-convex function $f\left(x_{1}, x_{2}\right)=\varphi\left(x_{1}-x_{2}\right)$ with $\varphi(t)$ in $(2.1)$, treated in Example 4.2, the modified discrete Hessian matrix (4.4) at $\left(x_{1}, x_{2}\right)=(0,0)$ is

$$
\tilde{H}_{f}(0,0)=\left[\begin{array}{cc}
-2 \varphi(0)+\varphi(-1)+\varphi(1) & 2 \varphi(0)-\varphi(-1)-\varphi(1) \\
2 \varphi(0)-\varphi(-1)-\varphi(1) & -2 \varphi(0)+\varphi(-1)+\varphi(1)
\end{array}\right]=\left[\begin{array}{rr}
2 & -2 \\
-2 & 2
\end{array}\right]
$$

This is positive semidefinite.

Example 4.5. For the $L^{\natural}$-convex function (4.3) in Example 4.3, the modified discrete Hessian matrix $(4.4)$ at $\left(x_{1}, x_{2}, x_{3}, x_{4}\right)=(0,0,0,0)$ is

$$
\tilde{H}_{f}(0,0,0,0)=\left[\begin{array}{rrrr}
1 & -1 & 0 & 0 \\
-1 & 1 & 0 & 0 \\
0 & 0 & 1 & -1 \\
0 & 0 & -1 & 1
\end{array}\right]
$$

This is positive semidefinite.

Convex extensibility alone does not imply positive semidefiniteness of the modified discrete Hessian $\tilde{H}_{f}(x)=\left(\tilde{H}_{i j}(x)\right)$ in $(4.4)$, which is demonstrated by Example 4.6 below. On the other hand, $\mathrm{L}^{\mathrm{q}}$-convexity, which is a combinatorial convexity concept, does imply both convex extensibility and positive semidefiniteness of $\tilde{H}_{f}(x)$ via (4.5) and (4.6).

Example 4.6. We define $f: \mathbb{Z}^{2} \rightarrow \mathbb{R}$ by $f\left(x_{1}, x_{2}\right)=\varphi\left(x_{1}+x_{2}\right)$ with the univariate convex function

$$
\varphi(t)= \begin{cases}|t|^{2} & (|t| \leq 1) \\ 2|t|-1 & (|t| \geq 1)\end{cases}
$$

The modified discrete Hessian matrix $\tilde{H}_{f}\left(x_{1}, x_{2}\right)$ of $f$ at $\left(x_{1}, x_{2}\right)=(0,0)$ is given by

$$
\tilde{H}_{f}(0,0)=\left[\begin{array}{ll}
\varphi(3)-2 \varphi(2)+\varphi(1) & \varphi(2)-2 \varphi(1)+\varphi(0) \\
\varphi(2)-2 \varphi(1)+\varphi(0) & \varphi(3)-2 \varphi(2)+\varphi(1)
\end{array}\right]=\left[\begin{array}{ll}
0 & 1 \\
1 & 0
\end{array}\right] .
$$

This is not positive semidefinite, whereas $f$ is convex extensible (not $L^{b}$-convex). 


\section{Mixed-Variable Functions}

Convexity concepts have also been discussed for functions $f: \mathbb{Z}^{n} \times \mathbb{R}^{m} \rightarrow \mathbb{R}$ with discrete and continuous variables $[2,7-10]$. In [8-10], to be specific, "mixed convexity" is discussed for functions $f: \mathbb{Z}^{n} \times \mathbb{R}^{m} \rightarrow \mathbb{R}$ with particular reference to the mixed Hessian matrix, which is defined as follows:

$$
H_{f}(x, y)=\left[\begin{array}{cc}
{\left[\nabla_{i j}(f(x, y))\right]_{n \times n}} & {\left[\nabla_{i}\left(\frac{\partial}{\partial y_{l}} f(x, y)\right)\right]_{n \times m}} \\
{\left[\frac{\partial}{\partial y_{k}}\left(\nabla_{j} f(x, y)\right)\right]_{m \times n}} & {\left[\frac{\partial^{2}}{\partial y_{k} \partial y_{l}} f(x, y)\right]_{m \times m}}
\end{array}\right]
$$

where

$$
\begin{aligned}
\nabla_{i j}(f(x, y)) & =f\left(x+e_{i}+e_{j}, y\right)-f\left(x+e_{i}, y\right)-f\left(x+e_{j}, y\right)+f(x, y), \\
\nabla_{i}\left(\frac{\partial}{\partial y_{l}} f(x, y)\right) & =\frac{\partial f}{\partial y_{l}}\left(x+e_{i}, y\right)-\frac{\partial f}{\partial y_{l}}(x, y), \\
\frac{\partial}{\partial y_{k}}\left(\nabla_{j} f(x, y)\right) & =\frac{\partial f}{\partial y_{k}}\left(x+e_{j}, y\right)-\frac{\partial f}{\partial y_{k}}(x, y), \\
\frac{\partial^{2}}{\partial y_{k} \partial y_{l}} f(x, y) & =\frac{\partial^{2} f}{\partial y_{k} \partial y_{l}}(x, y)
\end{aligned}
$$

and $\left[\nabla_{i j}(f(x, y))\right]_{n \times n}$ means the $n \times n$ matrix that has $\nabla_{i j}(f(x, y))$ as its $(i, j)$ entry, and similarly for $\left[\nabla_{i}\left(\frac{\partial}{\partial y_{l}} f(x, y)\right)\right]_{n \times m},\left[\frac{\partial}{\partial y_{k}}\left(\nabla_{j} f(x, y)\right)\right]_{m \times n}$, and $\left[\frac{\partial^{2}}{\partial y_{k} \partial y_{l}} f(x, y)\right]_{m \times m}$. See Remark 5.1 for some definitions and propositions in [10].

In this section we point out, by way of examples, that the positive semidefiniteness of the mixed Hessian matrix of $f$ is not implied by the convex extensibility of $f$.

Let $\varphi(t)$ be a univariate $\mathrm{C}^{2}$ convex function $\varphi: \mathbb{R} \rightarrow \mathbb{R}$. We define $f: \mathbb{Z} \times \mathbb{R} \rightarrow \mathbb{R}$ as $f(x, y)=\varphi(x-y)$. Such function $f$ is convex extensible, with a convex extension $\bar{f}$ given by $\bar{f}(x, y)=\varphi(x-y)$. According to the definition (5.1) we have

$$
H_{f}(0,0)=\left[\begin{array}{cc}
\varphi(2)-2 \varphi(1)+\varphi(0) & -\varphi^{\prime}(1)+\varphi^{\prime}(0) \\
-\varphi^{\prime}(1)+\varphi^{\prime}(0) & \varphi^{\prime \prime}(0)
\end{array}\right] .
$$

Example 5.1. In the case of $\varphi(t)=t^{4}$, we have $\varphi(0)=\varphi^{\prime}(0)=\varphi^{\prime \prime}(0)=0 ; \varphi(1)=1$, $\varphi^{\prime}(1)=4 ; \varphi(2)=16$ and the Hessian matrix (5.1) of $f$ is

$$
H_{f}(0,0)=\left[\begin{array}{rr}
14 & -4 \\
-4 & 0
\end{array}\right]
$$

This is not positive semidefinite in spite of the convex extensibility of $f$.

Example 5.2. In the case of $\varphi(t)$ of $(2.1)$, the Hessian matrix $(5.1)$ at $(0,0)$ is

$$
H_{f}(0,0)=\frac{1}{3}\left[\begin{array}{rr}
3 & -5 \\
-5 & 8
\end{array}\right]
$$

This is not positive semidefinite in spite of the convex extensibility of $f$.

The above examples show that the mixed Hessian matrix is not necessarily positive semidefinite even when $f$ is convex extensible.

Remark 5.1. Some definitions and propositions in [10] are reproduced here for critical comments. 
Definition 2. A mixed function $\Psi: \mathbb{Z}^{n} \times \mathbb{R}^{m} \rightarrow \mathbb{R}$ is called mixed convex if it is discretely convex with respect to its integer variables and convex with respect to its continuous variables.

Definition 3. A function is called strictly mixed convex if it is strictly discrete convex and strictly convex with respect to the continuous variables, simultaneously.

Definition 5. A mixed function is $k$-smooth if it is $k$-times differentiable (i.e. $C^{k}$ ) with respect to the real variables and if it can be differenced $k$-times with respect to the integer variables.

Theorem 4.4. A function $\Psi: \mathbb{Z}^{n} \times \mathbb{R}^{m} \rightarrow \mathbb{R}$ is 2 -smooth strictly mixed convex if and only if the mixed Hessian matrix for $\Psi$ is strictly positive.*

Corollary 5.1. $\Theta: \mathbb{Z} \times \mathbb{R} \rightarrow \mathbb{R}$ is a mixed convex function if and only if $\left.\Theta\right|_{\mathbb{Z}}$ is an integer convex function and $\left.\Theta\right|_{\mathbb{R}}$ is a real convex function.

Corollary 5.3. A function $\Theta: \mathbb{Z} \times \mathbb{R} \rightarrow \mathbb{R}$ is 2 -smooth strictly mixed convex if and only if $\Theta$ has a positive Hessian matrix.

As far as the present authors understand from the above definitions and Corollary 5.1, the function $\Theta: \mathbb{Z} \times \mathbb{R} \rightarrow \mathbb{R}$ defined by $\Theta(x, y)=x^{2} y^{2}$ for $x \in \mathbb{Z}$ and $y \in \mathbb{R}$ is mixed convex. The mixed Hessian matrix (5.1) at $(x, y)$ is given as

$$
H_{f}(x, y)=\left[\begin{array}{cc}
2 y^{2} & 2(2 x+1) y \\
2(2 x+1) y & 2 x^{2}
\end{array}\right] .
$$

We note that the diagonal entries are nonnegative, but the matrix $H$ is not positive semidefinite since its determinant $\operatorname{det} H_{f}(x, y)=-4 y^{2}\left(3 x^{2}+4 x+1\right)$ can be negative. It is also noted that the off-diagonal entries of $H$ are positive or negative depending on $(x, y)$. This seems to contradict Corollary 5.3 above.

\section{Conclusion}

We may summarize our observations as follows.

- Convex extensibility alone does not imply positive semidefiniteness of the discrete Hessian matrix (1.1). Counterexamples for $f: \mathbb{Z}^{2} \rightarrow \mathbb{R}$ have been given in Examples 2.1 and 2.2.

- Positive semidefiniteness of the discrete Hessian matrix (1.1) does not imply convex extensibility. A counterexample for $f: P(c) \rightarrow \mathbb{R}$ has been given in Section 3.1.2. Extensions of this function to $f: \mathbb{Z}_{+}^{2} \rightarrow \mathbb{R}$ and $f: \mathbb{Z}^{2} \rightarrow \mathbb{R}$ have been given in Sections 3.2 and 3.3 , respectively.

- $\mathrm{M}^{\mathrm{h}}$-convexity, which is a combinatorial convexity concept, does imply both convex extensibility and positive semidefiniteness of the discrete Hessian matrix in (1.1). Conversely, a certain combinatorial property of the discrete Hessian matrix, which is stronger than positive semidefiniteness, implies convex extensibility via $\mathrm{M}^{\natural}$-convexity (Theorem 4.1).

- $\mathrm{L}^{\natural}$-convexity, which is another combinatorial convexity concept, is not compatible with the discrete Hessian in (1.1), as shown in Examples 4.2 and 4.3. With the modified version of the discrete Hessian matrix in (4.4), $\mathrm{L}^{\natural}$-convexity does imply both convex extensibility and positive semidefiniteness of the (modified) discrete Hessian. Conversely, a certain combinatorial property of the (modified) discrete Hessian matrix, which is stronger than positive semidefiniteness, implies convex extensibility via $L^{\mathrm{h}}$-convexity (Theorem 4.2).

- Convex extensibility of a mixed function $f: \mathbb{Z}^{n} \times \mathbb{R}^{m} \rightarrow \mathbb{R}$ does not imply positive semidefiniteness of the discrete Hessian matrix (5.1). Counterexamples for $f: \mathbb{Z} \times \mathbb{R} \rightarrow \mathbb{R}$ have been given in Examples 5.1 and 5.2.

"An obvious typo " $\mathbb{R}^{n}$ " in $[10]$ is corrected to " $\mathbb{R}^{m}$ " here. 
The concepts of $M^{\natural}$-convexity and $L^{\natural}$-convexity for mixed functions $f: \mathbb{Z}^{n} \times \mathbb{R}^{m} \rightarrow \mathbb{R}$ are introduced in [2] and [7], respectively. The Hessian matrices of such functions are yet to be investigated.

\section{Acknowledgments}

This research is partially supported by KAKENHI $(21360045,22710148)$, the Global COE program "The Research and Training Center for New Development in Mathematics," and the Aihara Innovative Mathematical Modelling Project, the Japan Society for the Promotion of Science (JSPS) through the "Funding Program for World-Leading Innovative R\&D on Science and Technology (FIRST Program)," initiated by the Council for Science and Technology Policy (CSTP). The authors would like to thank Makoto Yamashita for his kind advice on an input data file of SDPA and anonymous referees for useful comments on a previous draft.

\section{References}

[1] H. Hirai and K. Murota: M-convex functions and tree metrics. Japan Journal of Industrial and Applied Mathematics, 21 (2004), 391-403.

[2] S. Moriguchi, S. Hara, and K. Murota: On continuous/discrete hybrid M ${ }^{\natural}$-convex functions. Transactions of the Institute of Systems, Control and Information Engineers, 20 (2007), 84-86 (in Japanese).

[3] S. Moriguchi and K. Murota: Discrete Hessian matrix for L-convex functions. IEICE Transactions on Fundamentals of Electronics, Communications and Computer Sciences, E88-A (2005), 1104-1108.

[4] S. Moriguchi and K. Murota: Constructing a nonconvex discrete function with positive semidefinite discrete Hessian matrix. METR 2011-36, Department of Mathematical Informatics, University of Tokyo (2011).

[5] K. Murota: Discrete Convex Analysis (SIAM, Philadelphia, 2003).

[6] K. Murota: Primer of Discrete Convex Analysis-Discrete versus Continuous Optimization (Kyoritsu Publishing Co., Tokyo, 2007) (in Japanese).

[7] Y. Takamatsu, S. Hara, and K. Murota: Continuous/discrete hybrid convex optimization and its optimality criterion. Transactions of the Institute of Systems, Control and Information Engineers, 17 (2004), 409-411 (in Japanese).

[8] E. Tokgöz: Mixed T-, T*-convex functions and their corresponding mixed Hessian matrices. International Journal of Pure and Applied Mathematics, 56 (2009), 133-141.

[9] E. Tokgöz: Algorithms for mixed convexity and optimization of 2-smooth mixed functions. International Journal of Pure and Applied Mathematics, 57 (2009), 103-110.

[10] E. Tokgöz, M. Maalouf, and H. Kumin: A Hessian matrix for functions with integer and continuous variables. International Journal of Pure and Applied Mathematics, $\mathbf{5 7}$ (2009), 209-218.

[11] M. Yamashita, K. Fujisawa, K. Nakata, M. Nakata, M. Fukuda, K. Kobayashi, and K. Goto: A high-performance software package for semidefinite programs: SDPA 7. Research Report B-460, Department of Mathematical and Computing Sciences, Tokyo Institute of Technology (2010). 
Satoko Moriguchi

School of Industrial Technology

Advanced Institute of Industrial Technology

1-10-40 Higashi-ohi, Shinagawa-ku

Tokyo 140-0011, Japan

E-mail: satokodaiit.ac.jp 